\title{
Ectopic expression of RASSF2 and its prognostic role for gastric adenocarcinoma patients
}

\author{
DENG LUO ${ }^{1}$, TING YE ${ }^{2}$, TIAN-QIAN LI ${ }^{2}$, PENG TANG $^{1}$, SHA-DONG MIN ${ }^{2}$, GONG-FANG ZHAO ${ }^{1}$, \\ HUA HUANG $^{1}$, JIANG CHANG ${ }^{1}$, YAN WANG $^{3}$, LIN LV $^{4}$, MING-LIANG LU ${ }^{1}$ and MENG-YAO ZHENG ${ }^{1}$ \\ ${ }^{1}$ Department of Gastroenterology, The Second Affiliated Hospital of Kunming Medical University; \\ ${ }^{2}$ Department of Oncology, Tumor Hospital of Yunnan Province, The Third Affiliated Hospital of \\ Kunming Medical University; ${ }^{3}$ Department of Pathology; ${ }^{4}$ Institute of Pharmacological Research, \\ The Second Affiliated Hospital of Kunming Medical University, Kunming, Yunnan, P.R. China
}

Received September 28, 2011; Accepted November 18, 2011

DOI: $10.3892 /$ etm.2011.440

\begin{abstract}
RASSF2 has recently been identified as a potential tumor suppressor that serves as a Ras effector in various types of human cancers. However, there have been few reports detailing this in gastric cancer. Samples of gastric adenocarcinoma from 276 Chinese patients with follow-up were analyzed for RASSF2 protein expression by immunohistochemistry. RASSF2 was expressed in up to $31.2 \%(86 / 276)$ of this group of gastric carcinoma. The expression of RASSF2 was significantly lower in carcinomas than in normal mucosas $(\mathrm{P}<0.05)$. RASSF2 corresponded positively with patient age, histological differentiation, depth of tumor invasion, regional lymph node and distant metastasis, and TNM stage (all $\mathrm{P}<0.05$ ). Further multivariate analysis revealed that patient gender, depth of tumor invasion, distant metastasis, TNM stage and the expression of RASSF2 were independent prognostic factors for patients with gastric cancer. The Kaplan-Meier plot showed that the overall mean survival time of the patients with RASSF2-negative expression was shorter than that of patients with positive expression $\left(\chi^{2}=156.874, \mathrm{P}<0.0001\right)$. Moreover, RASSF2-negative expression had a much more significant effect on the survival of those patients with early stage tumors $\left(\chi^{2}=127.167, \mathrm{P}<0.0001\right)$, highlighted by a $>50.9 \%$ reduction in 3 -year survival compared to that of patients with RASSF2positive expression. In late stages, the difference was also significant $\left(\chi^{2}=6.246, \mathrm{P}=0.019\right)$, with a $35.5 \%$ reduction in 3 -year survival. It is suggested that RASSF2 plays an important role in the evolution of gastric adenocarcinoma and should be considered as a potential marker for its prognosis.
\end{abstract}

Correspondence to: Dr Gong-Fang Zhao, Department of Gastroenterology, The Second Affiliated Hospital of Kunming Medical University, No. 1 MaYuan Road, Xishan, Kunming, Yunnan 650101, P.R. China

E-mail: 122068863@qq.com

Key words: RASSF2, gastric cancer, metastasis, prognosis

\section{Introduction}

By certain estimates, gastric cancer is the fourth most common type of cancer after lung, breast and colorectal, and the second leading cause of cancer-related death (1). Although its prognosis has improved in recent years, particularly in the West, gastric cancer remains a key public health issue in China $(2,3)$. In contrast to the patterns of incidence in the West, more new cases are diagnosed each year in China (4-7). However, gastric cancer is often diagnosed at an advanced stage. Increased evidence has indicated that gastric cancer results from various genetic and epigenetic alterations of oncogenes, tumorsuppressor genes, DNA repair genes, cell adhesion molecules and cell cycle regulators (8-10). Molecules involved in each step of the carcinogenesis process are potential prognostic and therapeutic markers.

The RASSF family of proteins consists of 10 members (RASSF1 to 10) with various isoforms, all of which share a region of homology, the ras association domain. Members of this family have been reported to suppress cell growth when expressed exogenously in cultured cells (11). The RASSF2 gene has been shown to be frequently inactivated by promoter methylation in a wide range of tumor types, including colorectal, gastric, oral, nasopharyngeal, breast and lung cancers (12-16). Further studies have identified that RASSF2 has a putative nuclear localization signal (NLS) and a nuclear export signal (NES) at the N- and C-terminal regions, respectively (17). In addition, RASSF2 acts in a complex manner that extends beyond simple protein-protein association to play an important role in MST1 regulation as RASSF2 protein stability is significantly decreased in the absence of MST1 in vitro and in vivo (18). To date, all evidence indicates that RASSF2 is a K-Ras-specific effector and potential tumor suppressor.

The present study was carried out to investigate alterations in the expression of RASSF2 in surgical specimens of gastric cancer, to explore the possible correlation between RASSF2 expression and clinicopathological variables, and to correlate the expression of RASSF2 with lymph node and distant metastasis. In addition, we also analyzed the prognostic significance of RASSF2 expression and assessed the impact of expression of the studied protein on patient survival. 


\section{Materials and methods}

Patients and tissue samples. This study included a total of 276 Chinese patients with primary gastric cancer. Gastric cancer tissues were obtained from gastrectomy specimens at the Department of Surgery and Pathology, The Second Affiliated Hospital of Kunming Medical University, from July 2000 to May 2006. Sixty-five non-cancerous human gastric tissues were obtained from gastrectomies of adjacent gastric cancer margins $>5 \mathrm{~cm}$. None of the patients had received chemotherapy or radiotherapy prior to surgery. Tissues were formalin-fixed, paraffin-embedded, and clinically and histopathologically diagnosed at the Departments of Gastrointestinal Surgery and Pathology. All patients had follow-up records for over 5 years. The follow-up deadline was March 2011. The survival time was determined from the date of surgery to the follow-up deadline or date of death, which was mostly caused by recurrence or metastasis. Clinicopathological findings were determined according to the TNM-7th edition 2009 (UICC/ AJCC) and Japanese Classification 2010 in Gastric Cancer $(19,20)$. There were 8 papillary adenocarcinomas, 187 tubular adenocarcinomas, 47 mucinous adenocarcinomas, 34 signet ring cell carcinomas and 17 highly differentiated adenocarcinomas; 90 were classified as moderately differentiated adenocarcinomas, 165 as poorly differentiated adenocarcinomas and 4 as undifferentiated adenocarcinomas or others. There were 32 cases with distant metastasis. Sixty cases were categorized as stage I, 97 were stage II, 86 were stage III and 33 were stage IV.

Immunohistochemistry of RASSF 2 in gastric cancer and its evaluation. According to the protocol for immunohistochemistry, on paraffin-embedded tissue sections, slides were baked at $60^{\circ} \mathrm{C}$ for $2 \mathrm{~h}$ followed by deparaffinization with xylene and rehydration. The sections were submerged into EDTA antigenic retrieval buffer and microwaved for antigenic retrieval, after which they were treated with $3 \%$ hydrogen peroxide in methanol to block endogenous peroxidase activity, followed by incubation with $1 \%$ bovine serum albumin to block non-specific binding. Sections were incubated with RASSF2 goat anti-human polyclonal antibody (LifeSpan Biosciences, USA) overnight at $4^{\circ} \mathrm{C}$. Normal goat serum was used as a negative control. After rinsing $2 \times 5 \mathrm{~min}$ with TBST, tissue sections were treated with a secondary antibody in TBS for $1 \mathrm{~h}$ at room temperature. Development with chromogen (DAB) at room temperature was observed under a microscope. Subsequently, all tissue sections were counterstained with hematoxylin, dehydrated and mounted. The nucleus with RASSF2 was stained as buffy, whereas weak expression was associated with the cytoplasm. Evaluation of immunohistochemistry was independently carried out by two investigators. In scoring the expression of RASSF2 protein, both the extent and intensity of immunopositivity were considered. The intensity of positivity was scored as follows: 0 , negative; 1 , weak; 2 , moderate; 3 , strong. The extent of positivity was scored according to the percentage of cells showing positive staining: $0,<5 \% ; 1,>5-25 \% ; 2,>25-50 \% ; 3,>50-75 \%$; $4,>75 \%$ of the cells in the respective lesions. The final score was determined by multiplying the intensity of positivity and the extent of positivity scores, yielding a range from 0 to 12 .
The expression for RASSF2 was considered positive when the scores were $\geq 5$.

Statistical analysis. Statistical analyses were performed with SPSS 16.0 software. The correlations among the expression of RASSF2 and clinicopathological characteristics were calculated by the Student's t-test and the Chi-square correlation test. The Kaplan-Meier method was used to estimate survival as a function of time, and survival differences were analyzed with the log-rank test. A multivariable test was performed to determine the factor correlated with survival length by Cox regression analysis. The statistical significance level was defined as $\mathrm{P}<0.05$.

\section{Results}

Expression of RASSF2 in gastric cancer and non-cancerous mucosa. RASSF2 was detected in $42(64.6 \%)$ of 65 human non-tumor mucosa. Positive expression of RASSF2 protein was detected in $31.2 \%$ (86/276) of 276 human gastric cancer cases, and negative expression was detected in 190 (68.8\%). RASSF2 staining was detected in the majority of normal cells, particularly in the nucleus and cytoplasm. We also found RASSF2-positive expression in intestinal metaplasia. However, RASSF2 was apparently down-expressed in the primary cancer. The differences in RASSF2 expression between gastric cancer and non-cancerous mucosa were also statistically significant $\left(\chi^{2}=21.115, \mathrm{P}<0.0001 ;\right.$ Fig. 1$)$.

RASSF2 expression and clinicopathological features. Positive expression of RASSF2 was correlated with patient age, histological differentiation, depth of tumor invasion, regional lymph node and distant metastasis, and TNM stage (all $\mathrm{P}<0.05$ ). RASSF2 expression did not correlate with patient gender, size of tumor and histological type ( $\mathrm{P}>0.05$; Table I). The factors with possible prognostic effects in gastric carcinoma were analyzed by Cox regression analysis. The study revealed that patient gender $(\mathrm{P}=0.003)$, depth of tumor invasion $(\mathrm{P}=0.040)$, distant metastasis $(\mathrm{P}<0.0001)$, TNM stage $(\mathrm{P}<0.0001)$ and the expression of RASSF2 $(\mathrm{P}<0.0001)$ were independent prognostic factors for patients with gastric carcinoma. However, patient age, tumor size, histological type, histological differentiation and regional lymph node metastasis had no prognostic value (Table II).

Correlation between RASSF2 expression and patient prognosis. The Kaplan-Meier plot showed that the survival time of patients with negative RASSF2 expression was significantly lower than that in patients with positive RASSF2 expression. The survival estimates showed a marked difference in median survival between patients with positive and negative RASSF2 expression; the former averaged 55 months (95\% CI 52.24-55.76), whereas the latter averaged 21 months (95\% CI 19.15-22.86). For patients with negative RASSF2 protein expression, the 1- and 3-year survival rate was 79.5 and $14.2 \%$, respectively, significantly lower than in patients with positive expression (97.3 and $89.3 \%$, respectively; $\left.\chi^{2}=156.874, \mathrm{P}<0.0001\right)$. From this result, we concluded that decreased expression of RASSF2 is a prognostic indicator of poor survival for patients with gastric cancer (Table III and Fig. 2). Additionally, we further compared the survival 

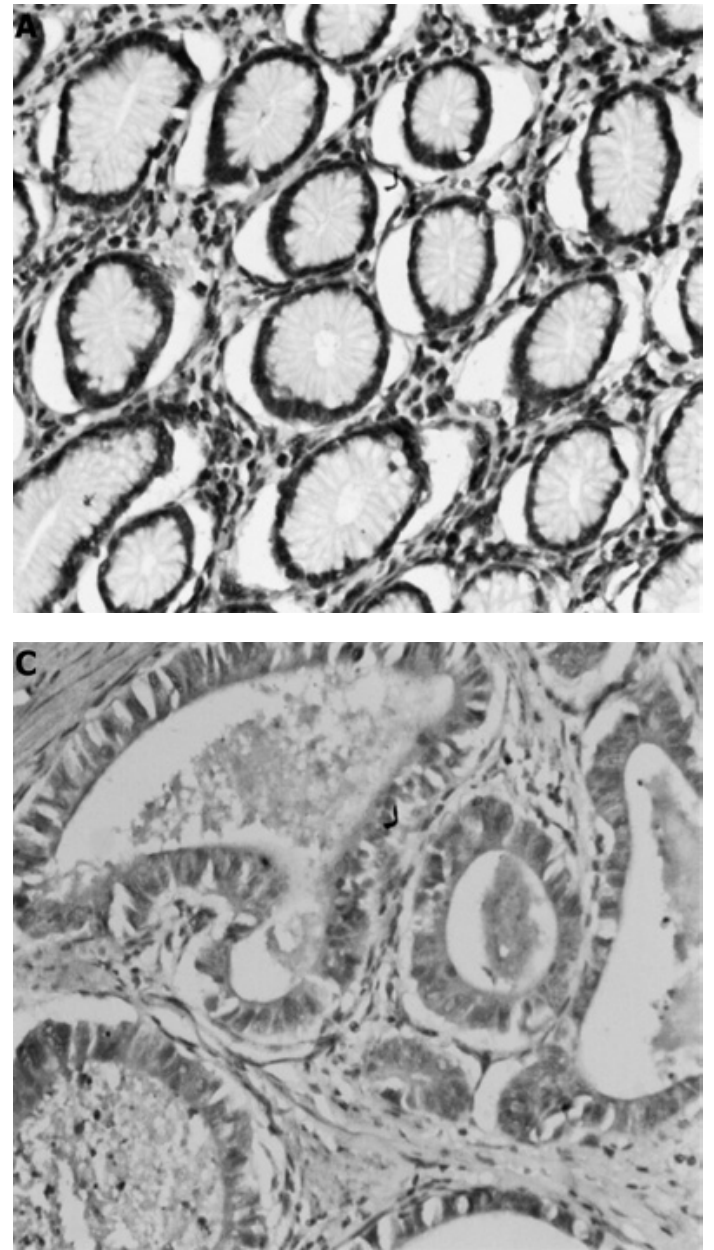
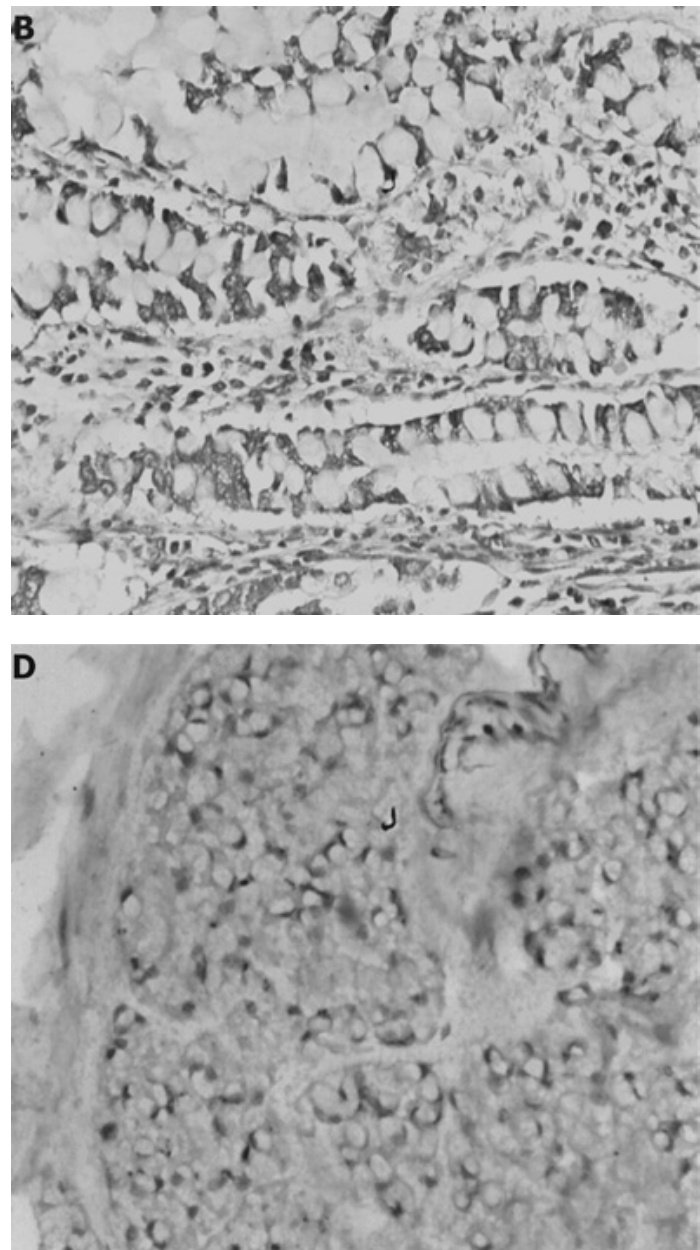

Figure 1. Immunohistochemical staining for RASSF2 in gastric cancer lesions and non-cancerous tissues. (A) RASSF2 was positively expressed in noncancerous tissues; (B) RASSF2 was also positively detected in intestinal metaplasia cells; (C) RASSF2 was negatively or weakly expressed in tubular adenocarcinoma; (D) RASSF2 staining was negative in Signet ring cell carcinoma. Magnification, x200.

times between the patients who differed in terms of RASSF2 expression, respectively, and who were in early TNM stage (I and II) or late TNM stage (III and IV). The results showed that RASSF2-negative expression had a much more significant effect on the survival of those patients with early-stage tumors $\left(\chi^{2}=127.167, \mathrm{P}<0.0001\right)$, highlighted by a $>50.9 \%$ reduction in 3-year survival compared to that of patients with RASSF2-positive expression. In late stages, the difference was also significant $\left(\chi^{2}=6.246, P=0.019\right)$, with a $35.5 \%$ reduction in 3-year survival. Notably, these data indicate that ectopic expression of RASSF2 is an independent prognostic variable for gastric cancer in early stage and late stage (Table III).

\section{Discussion}

Epigenetic inactivation of tumor-suppressor genes is a fundamental event in the development of many types of cancers $(21,22)$. Here, we demonstrated that reduced expression of RASSF2 frequently occured in gastric cancer lesions. Several previous studies have shown that down-regulation of RASSF2 by promoter hypermethylation occurs in different tumor cell lines and primary tumors, including lung, breast, colorectal, nasopharyngeal and thyroid cancer. RASSF2 inhibits the growth of tumor cells, and its growth-inhibitory properties are enhanced by activated K-Ras $(23,24)$. RASSF2 promotes both cell cycle arrest and apoptosis.

Akino et al (12) used RT-PCR and bisulfite PCR to analyze the expression and methylation status of six RASSF family genes in colorectal cancer cell lines and in primary colorectal cancers and colorectal adenomas. They found that aberrant methylation and histone deacetylation of RASSF2 were associated with the gene silencing in colorectal cancer. Primary colorectal cancers that showed K-ras/BRAF mutations also frequently showed RASSF2 methylation, and inactivation of RASSF2 enhanced K-ras-induced oncogenic transformation. Moreover, RASSF2 methylation was also frequently observed in colorectal adenomas. Finally, they concluded that RASSF2 is a novel tumor-suppressor gene that regulates Ras signaling and plays a critical role in the early stages of colorectal tumorigenesis.

On the other hand, Endoh et al (25) carried out the first detailed investigation and determined that frequent silencing of RASSF2 was associated with promoter hypermethylation in gastric cancer. Their study revealed that methylation frequencies of RASSF2 varied in the regions upstream and downstream of the transcription start site. They also observed that gastric cancers with methylation at U1 and D1 exhibited significantly less frequent lymphatic permeation than unmethylated gastric cancers. Afterwards, epigenetic inactivation of RASSF2 was 
Table I. Relationship of RASSF2 expression with pathological parameters of gastric cancer.

\begin{tabular}{|c|c|c|c|c|c|}
\hline \multirow[t]{2}{*}{ Clinical parameters } & \multirow[t]{2}{*}{$\mathrm{n}$} & \multicolumn{2}{|c|}{ RASSF2 } & \multirow[t]{2}{*}{$\chi^{2}$-test } & \multirow[t]{2}{*}{ P-value } \\
\hline & & Positive, n (\%) & Negative, n (\%) & & \\
\hline \multicolumn{6}{|l|}{ Age (years) } \\
\hline$<50$ & 100 & $40(40.0)$ & $60(60.0)$ & & \\
\hline$\geq 50$ & 176 & $46(26.1)$ & $130(73.9)$ & 5.714 & 0.0170 \\
\hline \multicolumn{6}{|l|}{ Gender } \\
\hline Male & 169 & $53(31.4)$ & $116(68.6)$ & & \\
\hline Female & 107 & $33(30.8)$ & $74(69.2)$ & 0.008 & 0.9280 \\
\hline \multicolumn{6}{|l|}{ Size (cm) } \\
\hline$<5$ & 137 & $36(26.3)$ & $101(73.7)$ & & \\
\hline$\geq 5$ & 139 & $50(36.0)$ & $89(64.0)$ & 3.023 & 0.0820 \\
\hline \multicolumn{6}{|l|}{ Histology } \\
\hline Papillary adenocarcinoma & 8 & $4(50.0)$ & $4(50.0)$ & & \\
\hline Tubular adenocarcinoma & 187 & $63(33.7)$ & $124(66.3)$ & & \\
\hline Mucinous adenocarcinoma & 47 & $11(23.4)$ & $36(76.6)$ & & \\
\hline Signet ring cell carcinoma & 34 & $8(23.5)$ & $26(76.5)$ & 4.123 & 0.2490 \\
\hline \multicolumn{6}{|l|}{ Histological differentiation } \\
\hline Well & 17 & $7(41.2)$ & $10(58.8)$ & & \\
\hline Moderate & 90 & $38(42.2)$ & $52(57.8)$ & & \\
\hline Poor & 165 & $38(23.0)$ & $127(77.0)$ & & \\
\hline Other & 4 & $1(25.0)$ & $3(75.0)$ & 9.680 & 0.0080 \\
\hline \multicolumn{6}{|l|}{ T stage } \\
\hline $\mathrm{T} 1$ & 21 & $19(90.5)$ & $2(9.5)$ & & \\
\hline $\mathrm{T} 2$ & 54 & $27(50.0)$ & $27(50.0)$ & & \\
\hline T3 & 163 & $38(23.3)$ & 125 (76.7) & & \\
\hline $\mathrm{T} 4 \mathrm{a}$ & 30 & $2(6.7)$ & $28(93.3)$ & & \\
\hline $\mathrm{T} 4 \mathrm{~b}$ & 8 & $2(25.0)$ & $6(75.0)$ & 59.941 & $<0.0001$ \\
\hline \multicolumn{6}{|l|}{$\mathrm{N}$ stage } \\
\hline N0 & 145 & $81(55.9)$ & $64(44.1)$ & & \\
\hline N1 & 35 & $2(5.7)$ & $33(94.3)$ & & \\
\hline $\mathrm{N} 2$ & 50 & $2(4.0)$ & $48(94.0)$ & & \\
\hline N3a & 22 & $3(13.6)$ & $19(86.4)$ & & \\
\hline $\mathrm{N} 3 \mathrm{~b}$ & 24 & $1 \quad(4.2)$ & $23(95.8)$ & 87.028 & $<0.0001$ \\
\hline \multicolumn{6}{|l|}{ M stage } \\
\hline M0 & 244 & $84(34.4)$ & $160(65.6)$ & & \\
\hline M1 & 32 & $2(6.3)$ & 30 (93.7) & 10.470 & 0.0010 \\
\hline \multicolumn{6}{|l|}{ TNM stage } \\
\hline I & 60 & $44(73.3)$ & $16(26.7)$ & & \\
\hline II & 97 & $36(37.1)$ & $61(62.9)$ & & \\
\hline III & 86 & $3(3.5)$ & 83 (96.5) & & \\
\hline IV & 33 & $3(9.1)$ & $30(90.9)$ & 93.519 & $<0.0001$ \\
\hline
\end{tabular}

${ }^{\mathrm{a}} \mathrm{AJCC} / \mathrm{UICC}, 7$ th edition.

Table II. Multivariate analysis with Cox proportional hazards model for prognostic factors.

\begin{tabular}{lccc}
\hline Factor & P-value & Hazard ratio & 95\% CI \\
\hline Gender & 0.0030 & 1.527 & $1.157-2.014$ \\
Depth of invasion & 0.0400 & 0.755 & $0.577-0.987$ \\
Distant metastasis & $<0.0001$ & 6.498 & $3.288-12.844$ \\
TNM stage & $<0.0001$ & 2.150 & $1.575-2.934$ \\
RASSF2 & $<0.0001$ & 0.129 & $0.082-0.202$ \\
\hline
\end{tabular}

found in oral squamous cell carcinoma (16), head and neck squamous cell carcinoma (26), hepatocellular (27) and thyroid cancers (28). Although a previous study demonstrated that expression of the RASSF2 gene is silenced by methylation in human gastric cancer (29), the authors did not clarify the clinical impact of RASSF2 expression or the prognostic value for patients with gastric cancer since the number of cases was too small. Thus, our study is the first to determine the correlation between RASSF2 expression and clinical and prognostic factors in gastric cancer. 
Table III. Survival estimates (and 95\% CIs) for disease-related deaths.

\begin{tabular}{lccccc}
\hline Group & Median survival (months) & 1-year survival rate & 3-year survival rate & $\chi^{2}$-test & P-value \\
\hline Positive & $55.0(52.24-55.76)$ & $73(97.3 \%)$ & $67(89.3 \%)$ & & $<0.0001$ \\
Negative & $21.0(19.15-22.86)$ & $140(79.5 \%)$ & $25(14.2 \%)$ & 156.874 & \\
Stage I-II & & & & & \\
Positive & $52.5(25.00-59.00)$ & $85(86.5 \%)$ & $81(82.6 \%)$ & & \\
Negative & $29.6(24.00-36.00)$ & $79(95.1 \%)$ & $27(31.7 \%)$ & & \\
Stage III-IV & & $7(77.9 \%)$ & $5(45.6 \%)$ & & 0.0001 \\
Positive & $31.0(2.50-39.50)$ & $79(67.8 \%)$ & $10(10.1 \%)$ & 6.246 & 0.0190 \\
Negative & $16.0(10.00-21.00)$ & & & &
\end{tabular}

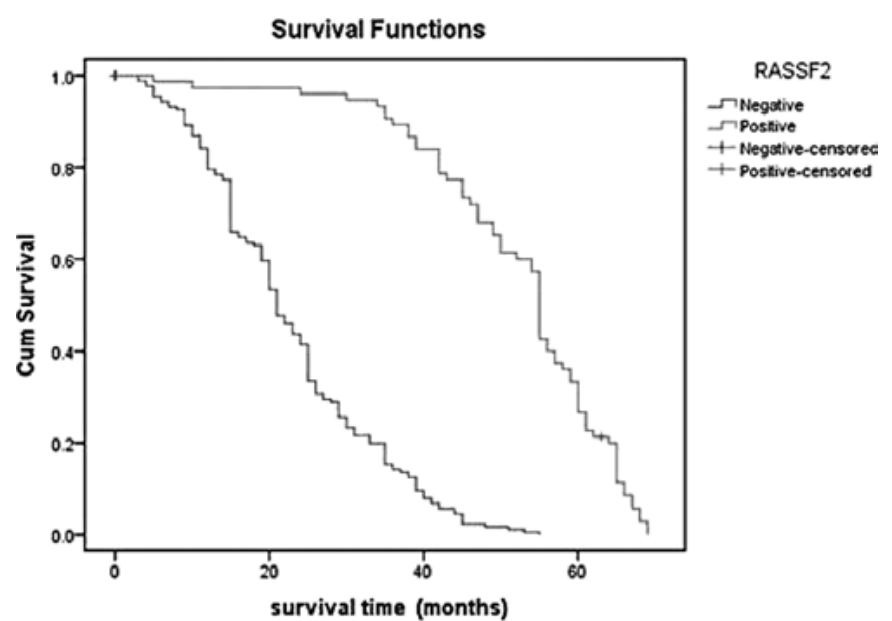

Figure 2. Kaplan-Meier curves with univariate analyses (log-rank) for patients with tumors with RASSF2-negative expression vs. RASSF2-positive expression for all gastric cancer cases. The cumulative 1- and 3-year survival rate was 97.3 and $89.3 \%$, respectively, in the positive RASSF2 protein expression group, but only 79.5 and $14.2 \%$ in the negative expression group $\left(\chi^{2}=156.874, \mathrm{P}<0.0001\right)$.

The present study demonstrated that RASSF2 expression was markedly down-regulated in gastric cancer tissues in comparison to that in normal gastric tissues. RASSF2 was detected in $42(64.6 \%)$ of 65 human non-tumor mucosa. However, positive expression of RASSF2 protein was detected in $31.2 \%$ (86/276) of 276 human gastric cancer cases, and negative expression was detected in 190 (68.8\%). Moreover, our data revealed that positive expression of RASSF2 was correlated with patient age, histological differentiation, depth of tumor invasion, regional lymph node and distant metastasis, and TNM stage. RASSF2 expression was not correlated with patient gender, size of the tumor and histological type. Further multivariate analysis revealed that patient gender, depth of invasion, distant metastasis, TNM stage and the expression of RASSF2 were independent prognostic factors for the disease. Additionally, the Kaplan-Meier plot showed that survival time of patients with negative RASSF2 expression was significantly lower compared to patients with positive RASSF2 expression. For patients with negative RASSF2 protein expression, the 1- and 3-year survival rate was 79.5 and $14.2 \%$, respectively, which was significantly lower compared to patients with positive expression (97.3 and 89.3\%, respectively).
In addition, the survival time between the patients who differed in terms of RASSF2 expression and who were in early TNM stage (I and II) or late TNM stage (III and IV) was compared. The results showed that RASSF2-negative expression had a much more significant effect on the survival of those patients with early-stage tumors, highlighted by a $>50.9 \%$ reduction in 3-year survival compared to that of patients with RASSF2-positive expression. In late stages, the difference was also significant, with a $35.5 \%$ reduction in 3-year survival. Therefore, all evidence suggests that RASSF2 expression is an independent prognostic variable for gastric cancer in early and late stage.

To sum up, the potentially important consequence of our study is that RASSF2 may be an attractive therapeutic candidate for gastric cancer, as negative RASSF2 expression is predictive of outcome in early-stage disease, and may also be a feasible target for early intervention and treatment. In view of this, routine detection of methylation of this gene in blood may have utility in monitoring and detecting tumor recurrence in early-stage gastric cancer after curative surgical resection. Thus, the studied protein has provided a basis for the development of a potential biomarker for diagnosis and a candidate for molecular-targeted therapeutics of gastric cancer.

\section{Acknowledgements}

This study was supported by research grants from the Health Technology Fund of Yunnan Province, China (no. 2010NS066). Thanks to ProteinTech Group for the antibody, and to Jiang Chang for the statistical work.

\section{References}

1. Jemal A, Siegel R, Ward E, et al: Cancer statistics, 2009. CA Cancer J Clin 59: 225-249, 2009.

2. Ye YW, Dong RZ, Zhou Y, et al: Prognostic analysis of familial gastric cancer in Chinese population. J Surg Oncol 104: 76-82, 2011.

3. Thun M, Jemal A, Desantis C, et al: An overview of the cancer burden for primary care physicians. Prim Care 36: 439-454, 2009.

4. Chen JG, Zhu J, Zhang YH and Lu JH: Cancer survival in Qidong, China, 1992-2000. IARC Sci Publ: 43-53, 2011.

5. Law SC and Mang OW: Cancer survival in Hong Kong SAR, China, 1996-2001. IARC Sci Publ: 33-41, 2011.

6. Xiang YB, Jin F and Gao YT: Cancer survival in Shanghai, China, 1992-1995. IARC Sci Publ: 55-68, 2011

7. Xishan $\mathrm{H}$, Chen $\mathrm{K}$, Min $\mathrm{H}$, et al: Cancer survival in Tianjin, China, 1991-1999. IARC Sci Publ: 69-84, 2011. 
8. Rodriguez-Paredes M and Esteller M: Cancer epigenetics reaches mainstream oncology. Nat Med 17: 330-339, 2011.

9. Watanabe $\mathrm{Y}$ and Maekawa M: Methylation of DNA in cancer. Adv Clin Chem 52: 145-167, 2010.

10. Hatziapostolou M and Iliopoulos D: Epigenetic aberrations during oncogenesis. Cell Mol Life Sci 68: 1681-1702, 2011.

11. Liu C, Pan Y, Wang X, et al: Activation of RASSF2A by $\mathrm{p} 300$ induces late apoptosis through histone hyperacetylation. Cell Biol Int 34: 1133-1139, 2010.

12. Akino K, Toyota M, Suzuki H, et al: The Ras effector RASSF2 is a novel tumor-suppressor gene in human colorectal cancer. Gastroenterology 129: 156-169, 2005.

13. Kaira K, Sunaga N, Tomizawa Y, et al: Epigenetic inactivation of the RAS-effector gene RASSF2 in lung cancers. Int J Oncol 31: $169-173,2007$.

14. Zhang Z, Sun D, Van do N, et al: Inactivation of RASSF2A by promoter methylation correlates with lymph node metastasis in nasopharyngeal carcinoma. Int J Cancer 120: 32-38, 2007.

15. Cooper WN, Dickinson RE, Dallol A, et al: Epigenetic regulation of the ras effector/tumour suppressor RASSF2 in breast and lung cancer. Oncogene 27: 1805-1811, 2008.

16. Imai T, Toyota M, Suzuki H, et al: Epigenetic inactivation of RASSF2 in oral squamous cell carcinoma. Cancer Sci 99: 958-966, 2008

17. Kumari G, Singhal PK, Rao MR and Mahalingam S: Nuclear transport of Ras-associated tumor suppressor proteins: different transport receptor binding specificities for arginine-rich nuclear targeting signals. J Mol Biol 367: 1294-1311, 2007.

18. Song $\mathrm{H}, \mathrm{Oh} \mathrm{S}, \mathrm{Oh} \mathrm{HJ}$ and Lim DS: Role of the tumor suppressor RASSF2 in regulation of MST1 kinase activity. Biochem Biophys Res Commun 391: 969-973, 2010.

19. Washington K: 7th edition of the AJCC cancer staging manual: stomach. Ann Surg Oncol 17: 3077-3079, 2010

20. Santiago JM, Sasako M and Osorio J: TNM-7th edition 2009 (UICC/AJCC) and Japanese Classification 2010 in Gastric Cancer. Towards simplicity and standardisation in the management of gastric cancer. Cir Esp 89: 275-281, 2011 (In Spanish).
21. Resende C, Ristimaki A and Machado JC: Genetic and epigenetic alteration in gastric carcinogenesis. Helicobacter 15 (Suppl 1): 34-39, 2010

22. Nobili S, Bruno L, Landini I, et al: Genomic and genetic alterations influence the progression of gastric cancer. World J Gastroenterol 17: 290-299, 2011.

23. Donninger H, Hesson L, Vos M, et al: The Ras effector RASSF2 controls the PAR-4 tumor suppressor. Mol Cell Biol 30: 2608-2620, 2010.

24. Hesson LB, Wilson R, Morton D, et al: CpG island promoter hypermethylation of a novel Ras-effector gene RASSF2A is an early event in colon carcinogenesis and correlates inversely with K-ras mutations. Oncogene 24: 3987-3994, 2005.

25. Endoh M, Tamura G, Honda T, et al: RASSF2, a potential tumour suppressor, is silenced by $\mathrm{CpG}$ island hypermethylation in gastric cancer. Br J Cancer 93: 1395-1399, 2005.

26. Steinmann K, Sandner A, Schagdarsurengin U and Dammann RH: Frequent promoter hypermethylation of tumorrelated genes in head and neck squamous cell carcinoma. Oncol Rep 22: 1519-1526, 2009.

27. Ren J, He W, Zhang R, et al: RASSF2A promoter methylation in hepatitis $\mathrm{B}$ virus-related hepatocellular carcinogenesis and its correlation with elevated serum alpha-fetoprotein level. J Huazhong Univ Sci Technolog Med Sci 29: 309-312, 2009.

28. Schagdarsurengin U, Richter AM, Hornung J, et al: Frequent epigenetic inactivation of RASSF2 in thyroid cancer and functional consequences. Mol Cancer 9: 264, 2010.

29. Maruyama R, Akino K, Toyota M, et al: Cytoplasmic RASSF2A is a proapoptotic mediator whose expression is epigenetically silenced in gastric cancer. Carcinogenesis 29: 1312-1318, 2008. 